\title{
Gastric cancer and the search for a good prognostic classification: a challenge
}

\section{Esther Uña Cidón}

Medical Oncology Service, Clinical University Hospital of Valladolid, Valladolid, Spain
Correspondence: Esther Uña Cidón Medical Oncology Service, Clinical University Hospital, C/Ramon y Cajal s/n, 47005 Valladolid, Spain Tel +34 678938050

Fax +34983420078

Email aunacid@hotmail.com
This article was published in the following Dove Press journal:

Clinical and Experimental Gastroenterology

21 July 2010

Number of times this article has been viewed

Introduction: Gastric cancer is the second most common cancer worldwide. The standard treatment is radical surgical resection, but $60 \%$ of patients will present recurrence. TNM classification (6th edition, American Joint Committee on Cancer) recommends the removal of at least 15 regional lymph nodes to carry out an adequate pathological staging. But in our environment, only $20 \%$ of cases have the minimum recommended, so it would be very important to have an alternative prognostic. We designed a retrospective study to evaluate different prognostic factors in patients inadequately staged.

Material and methods: We focused on patients with serosal involvement (pT3). The evaluation included general parameters such as age, sex, tumor site, histological type, type of gastrectomy, histological grade, number of nodes analyzed $</ \geq 10$, lymph node ratio (LNR) with a $20 \%$ cutoff value, and adjuvant treatment. The association between clinicopathological variables and recurrence was investigated by univariate and multivariate logistic regression. It was considered statistically significant with $P<0.05$.

Results: A total of 92 patients who met the criteria were studied. Median age 65 years; $68 \%$ men, 32\% women; median follow-up time for the overall population, 44 months (range 15-119 months); number of nodes analyzed, median 7 (range 0-14 nodes); recurrence in 59\%; median time to recurrence, 15 months (range 3-48 months); the cumulative risk of relapse at five years, $64 \%$. Multivariate statistical analysis showed that the $\operatorname{LNR}(P=0.03)$ and total number of nodes analyzed $</ \geq 10(P=0.04)$ were independent predictors for the risk of recurrence.

Conclusion: LNR and total number of nodes analyzed with a threshold of $10(</ \geq 10$ nodes analyzed) were independent predictors of recurrence in patients with gastric carcinoma pT3 and an insufficient number of nodes examined.

Keyword: pathological classification

\section{Introduction}

Gastric cancer is the second most common cancer worldwide. ${ }^{1}$ Although there has been a trend of a decline in its incidence and mortality during the last decades, gastric cancer is still a relevant health problem around the world. ${ }^{2}$

Much effort has been made to improve its survival rate, although not much has been accomplished yet. The only potentially curative treatment for gastric cancer is radical surgery. ${ }^{1}$ Despite this, $60 \%$ of all these patients will relapse after complete resection; ${ }^{3}$ this fact being the cause of its high mortality rate. Although one of the most important prognostic factors in this disease are the pathological staging after complete resection, considerable controversy has surrounded the concept of what defines an adequate lymphadenectomy for potentially curative treatment of gastric cancer. ${ }^{2}$ 
Based on available published data, at least a D1 lymphadenectomy is recommended. ${ }^{2}$ In patients where there is a suspicion of N2 nodes, a D2 resection should be advised and should be performed by surgeons experienced with this technique. $^{2}$ According to TNM Classification of Malignant Tumors (6th edition), ${ }^{4}$ at least 15 nodes should be removed and evaluated to carry out an adequate pathological staging and subsequently to correctly divide patients into different prognostic subgroups.

Despite the lack of evidence, it would seem more beneficial to apply treatments in an individualized way; so much effort has to be focused on the search for an effective adjuvant or preoperative therapy and to find the best prognostic factors to select better patients who are more likely to benefit from these treatments to improve cure rates.

Few studies have suggested the ratio of the number of involved lymph nodes among the overall lymph nodes removed as another independent prognostic factor, which is more important than the node status..$^{5-7}$

Despite all these recommendations, only $20 \%$ of cases in our environment have the advised minimum of lymph nodes examined to carry out an accurate staging. This means there exists a high rate of understaged gastric cancer with the negative prognostic and therapeutic impact this fact could have. ${ }^{8,9}$ In this controversial context it would be very important to have alternative prognostic factors more related to recurrence risk in these understaged cases. With this aim, we developed a joint effort with General Surgery and Radiotherapy Oncology Departments in order to identify other clinicopathological factors as predictive of recurrence in patients undergoing gastric carcinoma surgery with less than 15 nodes evaluated. We focused only on patients with serosal involvement (pT3) in the histopathologycal analysis, because this subgroup was the most frequent (more than 70\%) encountered between patients radically operated.

\section{Material and methods}

We designed a retrospective study with the aim described above. All patients with primary gastric adenocarcinoma were evaluated in this current study. Eligible patients were individuals older than 18 years of age, with citologically or histologically proved gastric adenocarcinoma who had undergone radical surgery R0 according to the International Union Against Cancer. All these patients had less than 15 nodes evaluated and wall depth of invasion was pT3 according to TNM (6th edition). Patients with metastatic disease were excluded from our study.

This study was carried out at the Valladolid Clinical University Hospital between 2003 and 2006. The clinicopathologic parameters such as age, sex, histologic type, grade of tumor site, type of gastrectomy, $</ \geq 10$ total lymph nodes evaluated, lymph node ratio (LNR, number of involved lymph nodes among the overall lymph nodes removed) keeping a $20 \%$ cutoff value and adjuvant treatment. All these data were evaluated by reviewing medical charts and pathologic records. This study was approved by the Research Committee of our institution.

The association between all these clinicopathologic variables and recurrence was investigated by univariate and multivariate logistic regression analyses. Results were considered statistically significant when $P$ values were less than 0.05. All statistical analyses were conducted using SPSS 8.0 software package.

\section{Results}

We included 92 patients in this study with a median age of 65 years (range $32-76$ years). $68 \%$ were males and $32 \%$ females. All of them were from this geographical area and white race.

The median follow-up time for the overall population was 44 months (range 15-119 months). The median number of nodes analyzed was 7 (range $0-14$ nodes). $20 \%$ of our population was N0, having 0 nodes analyzed the $14 \%$ of them.

$64 \%$ of these patients didn't receive any adjuvant treatment (see Table 1).

Recurrence was detected in $59 \%$ of patients (total 54 patients) with a median time to relapse of 15 months (range

Table I Basal characteristics of included patients

\begin{tabular}{llll}
\hline & & $\mathbf{N}$ & $\%$ \\
\hline Sex & Male & 62 & 68 \\
Lauren type & Female & 30 & 32 \\
& Intestinal & 55 & 59 \\
Nodes & Diffuse & 37 & 41 \\
& Median & 7 & \\
Stage & Range & $0-14$ & \\
& O nodes analysed & 13 & \\
\multirow{4}{*}{ Age } & II (T3N0) & 20 & 22 \\
& III-A (T3NI) & 26 & 28 \\
Grade & III-B (T3N2) & 46 & 50 \\
& Median & 65 years & \\
& Range & $32-76$ years & \\
Location & I & 17 & 18 \\
& 2 & 34 & 36 \\
Adjuvant treatment & Cardia/fundus & 34 & 46 \\
& Stomach & 58 & 37 \\
& No & 59 & 63 \\
& Chemotherapy & 10 & 64 \\
& Chemoradiotherapy & 23 & 11 \\
\hline
\end{tabular}


3-48 months). 11 of them were locoregional recurrences,

7 cases presented distance metastases without locoregional relapse, and 36 showed both types of recurrences at the same time (see Table 2).

$77 \%$ of recurrences occurred within the first 2 years after surgical treatment. The estimated cumulative risk of relapse at 5 years in our study population was $64 \%$, which ranged between $85 \%$ and $46 \%$ respectively according to the LNR $>$ and $\leq 20 \%$.

The univariate and multivariate statistical analysis revealed that the LNR (odds ratio [OR] 2.14, 95\% confidence interval $[\mathrm{CI}]: 1.02-5.58, P=0.03)$ and total number of nodes analyzed $</ \geq 10$ (OR 2.75, 95\% CI: 1.5-8.21, $P=0.04)$ were independent predictors of recurrence in these patients.

\section{Discussion}

Gastric cancer still represents one of the most important causes of cancer-related morbidity and mortality around the world. ${ }^{2}$ Although much effort has been made to improve its prognosis, it remains with a high mortality rate which seems difficult to reduce. ${ }^{2}$

Many studies indicate that nodal involvement is one of the most important factors in determining the prognosis of patients with gastric carcinoma undergoing radical surgery, and it is significantly associated with the submucosal and serosal invasion of tumor cells. ${ }^{8}$ American Joint Committee on Cancer/Union International against cancer (AJCC/ UICC) TNM classification (6th edition) recommends the evaluation of at least 15 nodes to achieve an optimal staging. ${ }^{4}$ This is essential to accurately establish prognostic subgroups; however, in current clinical practice most cases could be understaged due to an inadequate number of lymph nodes retrieved. This fact has lead to search more accurate prognostic factors to better target the multidisciplinary therapy of these patients.

Previous studies have suggested that the LNR, which is defined as the ratio of the number of metastatic lymph

Table 2 Recurrences in our study population

\begin{tabular}{ll}
\hline & Total (\%) \\
\hline Recurrence & $54(64 \%)$ \\
Time to recurrence & \\
Median & 15 months \\
Range & $3-48$ months \\
Type of recurrence & \\
Locoregional & II (20\%) \\
Distance & $7(14 \%)$ \\
Both & $36(66 \%)$ \\
\hline
\end{tabular}

nodes to the total number of nodes dissected, is a powerful independent prognostic factor with significant superiority over more traditional factors. ${ }^{3,5-7}$ Although those studies have reported different cutoff values for LNR without having reached consensus, the most common one has been $20 \%$. In all these studies, an extended lymphadenectomy (D2), ${ }^{2}$ which is associated with a higher number of lymph nodes removed, was used as the standard surgical procedure; therefore, the issue of the prognostic value of the $\mathrm{N}$ ratio after $\mathrm{D} 1 \mathrm{lymph}$ node dissection has not been widely addressed. In the study by Marchet et $\mathrm{al}^{10}$ which addressed this point, the authors have concluded that $\mathrm{N}$ ratio is a simple and reproducible prognostic tool that can stratify patients with gastric cancer, also in case of limited lymph node dissection. These data may represent a good rationale for improving the prognostic power of current UICC TNM staging system and ultimately the selection of patients who may most benefit from adjuvant treatments.

In our institution we have previously carried out another study with the aim of evaluating different predictive factors for recurrence in patients inadequately staged, and we obtained a significant relationship between the depth of tumor involvement/LNR and the recurrence risk. ${ }^{9}$

Encouraged by these results and since more than $70 \%$ of our patients have serosal involvement (traditionally considered T3), we have decided to also evaluate the different clinicopathologic parameters and their relationship with the recurrence risk in this population.

We have studied 92 patients that had undergone a radical surgery in our institution. Although all of them had less than 15 nodes evaluated we obtained interesting results. The total number of nodes analyzed with a threshold of 10 $(</ \geq 10$ nodes analyzed) was an independent predictor of recurrence in these patients. We have decided the threshold of 10 nodes based on our previous study evaluating the clinicopathologic predictors of nodal metastases in patients undergoing radical resection. It is known that the higher the number of examined nodes, the higher the likelihood of involvement. Our results showed that invasion depth of tumor and number of lymph nodes examined $(</ \geq 6$ and $</ \geq 10)$ were independent predictors of nodal metastases. ${ }^{11}$ Based on these findings we tried to evaluate the same threshold in this specific population (T3).

On the other hand we have also shown the relevance of LNR in these patients as a predictor of recurrence. ${ }^{11}$

Although there have been controversial findings related to the $\mathrm{N}$ ratio in different Western and Japanese series, this factor has been considered as an effective prognostic tool after D2 or D3 lymphadenectomy. ${ }^{12-18}$ In a study by Bando et al 
at multivariate analysis the $\mathrm{N}$ ratio was retained as the only independent prognostic factor when the number and the side of metastatic lymph nodes were considered, ${ }^{13}$ and similarly, Kunisaki et al recently reported that the $\mathrm{N}$ ratio independently influenced the prognosis of 758 patients radically resected. ${ }^{19}$

In this way, the majority of the published studies addressing the prognostic value of $\mathrm{N}$ ratio in patients with gastric cancer have considered only patients who underwent extended lymphadenectomy (and therefore more than 15 lymph nodes dissected), so the study by Marchet et $\mathrm{al}^{10}$ is very important because it is the first report evaluating and demonstrating the prognostic power of $\mathrm{N}$ ratio among patients with inadequate lymph node dissection.

Unlike the $\mathrm{N}$ classification, whose prognostic power is significantly affected by the number of removed lymph nodes, the $\mathrm{N}$ ratio could distinguish subsets of patients with similar prognosis as the study by Marchet et al has demonstrated.

Overall, all these findings underline the ability of $\mathrm{N}$ ratio classification to identify prognostically homogeneous subsets of patients. Despite these findings, the new TNM classification ( 7 th edition) ${ }^{20}$ has suffered some modifications. Nowadays the old T3 has changed to T4a (a more advanced category), and $\mathrm{N}$ classification has been divided into 4 categories, although it continues considering just the number of nodes affected without taking into account the number of lymph nodes removed. Despite these changes more related with different prognostic subgroups, this new classification does not solve the problem of how to deal with patients inadequately staged. In these cases we think it would be relevant to consider the alternative of LNR to select patients according to recurrence risk.

\section{Disclosure}

The author reports no conflicts of interest in this work.

\section{References}

1. Cruz Hernández JJ, Rodriguez Sanchez CH. Lecciones de Oncología Clínica, 3rd ed. Sidonia, Spain: Nova Sidonia; 2004.

2. Catalano V, Labianca R, Beretta GD, et al. Gastric cancer. Crit Rev Oncol Hematol. 2009;71:127-164.

3. Xu DZ, Geng QR, Long ZJ, et al. Positive lymph node ratio is an independent prognostic factor in gastric cancer after D2 resection regardless of the examined number of lymph nodes. Ann Surg Oncol. 2009;16(2):319-326.

Clinical and Experimental Gastroenterology

\section{Publish your work in this journal}

Clinical and Experimental Gastroenterology is an international, peerreviewed, open access journal, publishing all aspects of gastroenterology in the clinic and laboratory, including: Pathology, pathophysiology of gastrointestinal disease; Investigation and treatment of gastointestinal disease; Pharmacology of drugs used in the alimentary tract;
4. International Union Against Cancer. In: Sobin LH, Wittekind CH, editors. TNM Classification of Malignant Tumours. 6th ed. New York, NY: Wiley-Liss Book; 2002.

5. Persiani R, Rausei S, Biondi A, et al. Ratio of metastatic lymph nodes: impact on staging and survival of gastric cancer. Eur J Surg Oncol. 2008;34(5):519-524.

6. Sun Z, Zhu GL, Lu C, Guo PT, Huang BJ, Li K, et al. The impact of $\mathrm{N}$-ratio in minimizing stage migration phenomenon in gastric cancer patients with insufficient number or level of lymph node retrieved: results from a Chinese monkey-institutional study in 2159 patients. Ann Oncol. 2009;20(5):897-905.

7. Xu DZ, Geng QR, Long ZJ, et al. Positive lymph node ratio is an independent prognostic factor in gastric cancer after D2 resection regardless of the examined number of lymph nodes. Ann Surg Oncol. 2009;16(2):319-326.

8. Siewert JR, Bottcher K, Stein HJ, Roder JD. Relevant prognostic factors in gastric cancer: ten-year results of the German Gastric Cancer Study. Ann Surg. 1998;228:449-461.

9. Uña E. Gastric cancer: predictors of recurrence when lymph-node dissection is inadequate. World J Surg Oncol. 2009;7:69.

10. Marchet A, Mocellin S, Ambrosi A, et al. The ratio between metastatic and examined lymph nodes ( $\mathrm{N}$ ratio) is an independent prognostic factor in gastric cancer regardless of the type of lymphadenectomy results from an Italian multicentric study in 1853 patients. Ann Surg. 2007;245(4):543-552.

11. Uña E, Fernández G, Torre A, et al. Predictive factors of lymph nodes metastases in gastric cancer. Ann Oncol. 2007;18 Suppl 7.

12. Bunt AMG, Hermans J, Smit VTHBM, et al. Surgical/pathologic-stage migration confronts comparisons of gastric cancer survival rate between Japan and Western countries. J Clin Oncol. 1995;13:19-25.

13. Bando E, Yonemura Y, Taniguchi K, et al. Outcome of ratio of lymph node metastasis in gastric carcinoma. Ann Surg Oncol. 2002;9: 775-784.

14. Inoue $\mathrm{K}$, Nakane $\mathrm{Y}$, Iiyama $\mathrm{H}$, et al. The superiority of ratio-based lymph node staging in gastric carcinoma. Ann Surg Oncol. 2002;9:27-34.

15. Nitti D, Marchet A, Olivieri M, et al. Ratio between metastatic and examined lymph nodes is an independent prognostic factor after D2 resection for gastric cancer: analysis of a large European monoinstitutional experience. Ann Surg Oncol. 2003;10:1077-1085.

16. Hyung WJ, Noh SH, Yoo $\mathrm{CH}$, et al. Prognostic significance of metastatic lymph node ratio in T3 gastric cancer. World J Surg. 2002;26: 323-329.

17. Kodera Y, Yamamura Y, Shimizu Y, et al. Lymph node status assessment for gastric carcinoma: is the number of metastatic lymph nodes really practical as a parameter for $\mathrm{N}$ categories in the TNM classification? J Surg Oncol. 1998;69:15-20.

18. Cheong JH, Hyung WJ, Shen JG, et al. The N ratio predicts recurrence and poor prognosis in patients with node-positive early gastric cancer. Ann Surg Oncol. 2006;13:377-385.

19. Kunisaki C, Shimada H, Nomura M, et al. Clinical impact of metastatic lymph node ratio in advanced gastric cancer. Anticancer Res. 2005;25: 1369-1376.

20. International Union Against Cancer. In: Sobin LH, Wittekind CH, Gospodarowicz M, editors. TNM Classification of Malignant Tumours. 7th ed. New York, NY: Wiley-Liss Book; 2009.

\section{Dovepress}

Immunology/genetics/genomics related to gastrointestinal disease. This journal is indexed on CAS. The manuscript management system is completely online and includes a very quick and fair peer-review system. Visit http://www.dovepress.com/testimonials.php to read real quotes from published authors. 